\title{
Deposition of silica protected luminescent layers of Eu:GdVO nanoparticles assisted by atmospheric pressure plasma jet
}

\author{
Elisa Moretti ${ }^{\mathrm{a}, *}$, Giorgia Pizzol $^{\mathrm{a}}$, Marina Fantin ${ }^{\mathrm{b}}$, Francesco Enrichi ${ }^{\mathrm{b}}$, Paolo Scopece ${ }^{\mathrm{b}}$, Nuria O. Nuñez $^{\mathrm{c}}$, \\ Manuel Ocaña ${ }^{c}$, Alvise Benedetti ${ }^{a}$, Stefano Polizzi ${ }^{\mathrm{a}, \mathrm{d}}$ \\ a Dipartimento di Scienze Molecolari e Nanosistemi, Università Ca' Foscari Venezia, INSTM Venice Research Unit, Via Torino 155/B, 30172 Mestre, Venezia, Italy \\ b Nanofab-Veneto Nanotech, Via delle Industrie 5, 30175 Marghera, Venezia, Italy \\ c Instituto de Ciencia de Materiales de Sevilla, CSIC-US, Americo Vespucio 49, 41092, Isla de la Cartuja, Sevilla, Spain \\ ' Centro di Microscopia Elettronica “Giovanni Stevanato”, Università Ca' Foscari Venezia, Via Torino 155/B, 30172 Mestre, Venezia, Italy
}

\section{A R T I C L E I N F O}

Article history:

Received 23 July 2015

Received in revised form 27 October 2015

Accepted 24 November 2015

Available online 18 December 2015

\section{Keywords:}

Eu-doped nanophosphors

Identification markers

Atmospheric pressure plasma jet

APPJ

Silica coating

\begin{abstract}
A B S T R A C T
Eu:GdVO ${ }_{4}$ nanophosphors with an average size of $60 \mathrm{~nm}$, synthesized by a facile solvothermal method, were deposited on monocrystalline silicon wafers by a spray-coating technique with artworks anti-counterfeiting applications in mind. Atmospheric pressure plasma jet (APPJ) was used to deposit a silica-based layer on top of the nanometric luminescent layer, in order to improve its adhesion to the substrate and to protect it from the environment. The nanophosphors were characterized by X-ray diffraction (XRD) and transmission electron microscopy (TEM). Coating composition was investigated by Fourier transform infrared spectroscopy (FT-IR) and its morphology was characterized by scanning electron microscopy (FEG-SEM). The film thickness was evaluated by means of ellipsometry and adhesion was estimated by a peeling test. Luminescent properties of the nanophosphors deposited and fixed on silicon wafers were also measured. The whole layer resulted well-adhered to the silicon substrate, transparent and undetectable in the presence of visible light, but easily activated by UV light source.
\end{abstract}

@ 2015 Published by Elsevier B.V.

\section{Introduction}

The design and characterization of new luminescent materials (also called phosphors) have gained enormous attention in the last years due to their wide potential in an incredible range of applications, from lighting to detectors, from displays to biolabels.

In some applications they are used in the form of a powder coating, patterned or not, like in monitors and fluorescent lamps. Luminescent materials produced by conventional methods usually consist of heterogeneous particles in the micrometre range; some applications, however, take advantage of a size in the nanometre range [1], for example for biolabeling or high-resolution displays. Due to their sub-wavelength size, such luminescent particles do not diffuse visible light and may be therefore used for optically transparent coatings. Such materials may find applications in phosphor-converted LEDs, identification marker for different goods, anti-counterfeiting label for artworks or as spectral converters for solar cells.

Among them, lanthanide-based nanoparticles (Ln-NPs) constitute an important and very promising new class of nanophosphors, since they combine the interesting optical properties of the doping ions and the advantageous features of nanoparticle matrix [2]. The use of highly luminescent rare-earth doped nanoparticles is successful for a large

\footnotetext{
* Corresponding author.

E-mail address: elisa.moretti@unive.it (E. Moretti).
}

number of reasons, including large Stokes' shifts, long-lived excited state lifetimes ( $1 \mathrm{~ms})$, higher photostability and quantum yields and lower toxicity compared to organic fluorophores [3]. Moreover a large palette of colours can be easily obtained by a convenient choice of the lanthanide ions [4]. In contrast to quantum dot, the colour of the emitted light does not depend on the size of the fluorescent nanoparticles, but only on the nature of $\mathrm{Ln}^{3+}$ ions.

Among the different luminescent compounds, those based on $\mathrm{Gd}$ orthovanadate $\left(\mathrm{GdVO}_{4}\right)$ are of special interest from the optical point of view, because the indirect excitation of the doping Ln cations through an energy transfer from the vanadate anion is much more efficient than the direct excitation of the Ln electronic levels, which results in a higher luminescence [5].

Depending on the field of application, luminescent coatings of the phosphors may be obtained by different methods like gravitational settling [6,7], electrophoretic deposition [8,9], Doctor Blade [10], spray methods [11,12], sol-gel coating [13-22], screen or ink-jet printing [23], Langmuir-Blodget technique [24] and others. Some of these methods use a binder, others a liquid media and some are dry methods. When particles are dispersed in a low-boiling liquid, once the latter has evaporated, the adhesion of the nanophosphors to the substrate is an important issue. In order to tackle this issue, particles and/or the surface substrate may be functionalised creating a chemical linkage between the coating layer and the substrate. A different physical approach is that of covering the nanoparticles with a fixation layer, which may 
also act as protecting layer. Two conditions must be satisfied for this method to be effective: a) the coating layer needs not to be compact, so as to leave islands of uncovered substrate where the fixation layer can anchor and $b$ ) the molecules of the fixation layer must be able to build a chemical link to the substrate. Thickness and homogeneity of the nanophosphors layer must be consequently taken under control. For these purposes, silica could behave as the ideal protective layer, because it has been shown to build up a transparent compact coating with a good anchorage to the substrate [25].

In the present work, a spray technique was chosen to realize a nanometric luminescent layer by depositing Eu-doped $\mathrm{GdVO}_{4}$ nanophosphors, with a narrow size distribution in the range of $60 \mathrm{~nm}$, on the surface of monocrystalline silicon wafers. Atmospheric pressure plasma jet (APPJ) was used to deposit a silica-based layer on top of the wafer for protecting the luminescent layer and improving its adhesion to the substrate, demonstrating that APPJ technology is a facile and efficient method to solve the problem of adhesion of nanoparticles deposited from a low-boiling liquid.

\section{Experimental}

\subsection{Reagents}

Gadolinium(III) nitrate hexahydrate $\left(\mathrm{GdNO}_{3} \cdot 6 \mathrm{H}_{2} \mathrm{O}\right.$, Aldrich, $\left.99.9 \%\right)$ and europium(III) nitrate pentahydrate $\left(\mathrm{Eu}\left(\mathrm{NO}_{3}\right)_{3} \cdot 5 \mathrm{H}_{2} \mathrm{O}\right.$, Aldrich, 99.9\%) were selected as Ln precursors. Sodium ortho-vanadate $\left(\mathrm{Na}_{3} \mathrm{VO}_{4}\right.$, Aldrich, $\left.99.98 \%\right)$ was used as a vanadium source. Ethylene glycol (EG, Acros, 99+\%), milliQ water and ethanol (EtOH, VWR Prolabo, 99.8\%) were used as solvents. Hexamethyldisiloxane (HMDSO, Aldrich, $\geq 98 \%$ ) was used as a chemical precursor for the APPJ silica deposition. Monocrystalline silicon wafers (Siegert Wafer $\mathrm{GmbH}$, resistivity 10$20 \Omega \cdot \mathrm{cm}$, thickness $525 \pm 20 \mu \mathrm{m}, 2.0 \times 2.5 \mathrm{~cm}$ ) were used as substrate for the deposition and coating processes.

\subsection{Preparation of Eu: $\mathrm{GdVO}_{4}$}

Nanoparticles of $5 \mathrm{~mol}$ \% Eu-doped Gd orthovanadate were prepared according to the procedure described by Nuñez et al. [26]. Gd (III)nitrate $\left(9.5 \cdot 10^{-2} \mathrm{mmol}\right)$ and $\mathrm{Eu}(\mathrm{III})$ nitrate $\left(5 \cdot 10^{3} \mathrm{mmol}\right)$ were dissolved in ethylene glycol $(2.5 \mathrm{~mL})$. In a separate vial, $\mathrm{Na}_{3} \mathrm{VO}_{4}(92.0 \mathrm{mg})$ was dissolved in an EG $(1.0 \mathrm{~mL})$ and milliQ $\mathrm{H}_{2} \mathrm{O}(1.5 \mathrm{~mL})$ mixture, then heated at around $80{ }^{\circ} \mathrm{C}$ under magnetic stirring to facilitate the dissolution of reagents. After cooling to room temperature, both solutions were mixed and the resulting solution was aged for $3 \mathrm{~h}$ in a tightly closed test tube at $120^{\circ} \mathrm{C}$. After ageing, the resulting dispersion was cooled down to room temperature, centrifuged to remove the supernatants and washed twice with ethanol and once with milliQ water. Finally, the precipitate was redispersed in ethanol and divided into portions to obtain different weight percentage of colloidal solutions, named Solxx, where $x x$ stands for the weight percent of nanoparticles in ethanol (e.g. Sol25 stands for a 0.25 wt.\% solution).

\subsection{Nanophosphor deposition and silica coating}

The $\mathrm{Eu}^{3+}$ doped $\mathrm{GdVO}_{4}$ nanoparticles were deposited by spray coating, and fixed to the substrate by applying a silica-based coating by means of an atmospheric pressure plasma jet system (Openair ${ }^{\circledR}$ Plasmatreater AS400 provided by Plasmatreat $\mathrm{GmbH}$ ).

For the spray process, the Eu-doped nanoparticles were dispersed in ethanol (with two different nanophosphor concentrations, $0.25 \%$ and $0.50 \%$ ) and sonicated for $15 \mathrm{~min}$; then a syringe pump pushed the ethanol solution into a coaxial nebulizer, which scanned the sample surface thanks to an $\mathrm{x}-\mathrm{y}$ automatic plotter. In order to obtain a more compact and less dusty film, the silicon wafer, previously cleaned with piranha solution $\left(\mathrm{H}_{2} \mathrm{SO}_{4}: \mathrm{H}_{2} \mathrm{O}_{2} 7: 3 \mathrm{vol}\right.$.), was heated at about $60{ }^{\circ} \mathrm{C}$ during the deposition (see ref. [27] for a schematic representation of the apparatus).
Following preliminary tests, the operating parameters were chosen as follows: $2 \mathrm{~mL} / \mathrm{min}$ (syringe flow rate), 3 bar (compressed air pressure), $10 \mathrm{~m} / \mathrm{min}$ (nozzle speed) and $90 \mathrm{~mm}$ (nozzle height). For the number of scans used $(4,6,8)$, see the discussion in Section 3 .

After spray deposition, the luminescent film was encapsulated in a silica-based coating film obtained via APPJ deposition using hexamethyldisiloxane (HMDSO) as a chemical precursor. The precursor was vaporized at $125^{\circ} \mathrm{C}$ and pushed towards the exit of the nozzle by means of a pressurized carrier gas. Following preliminary tests, the operating parameters were chosen as follows: 4 scans, $10 \mathrm{~g} / \mathrm{h}$ (evaporator HMDSO), $\mathrm{N}_{2}$ (ionization gas), Ar (carrier gas), $1500 \mathrm{~m} / \mathrm{min}$ (nozzle speed) and $12 \mathrm{~mm}$ (nozzle height).

The samples without silica coating will be referred in the following as "as-deposited", whereas the coated one will be referred as "fixed".

\subsection{Characterization techniques}

X-ray diffraction pattern (XRD) of the nanoparticles was obtained using a Philips X'Pert system (Bragg-Brentano parafocusing geometry) with a nickel-filtered $\mathrm{Cu} K \alpha 1$ radiation $(\lambda=0.154184 \mathrm{~nm}$ ).

Size and morphology of the nanoparticles were studied through a JEOL JEM 3010 transmission electron microscope (TEM) operating at $300 \mathrm{kV}$. The powder specimens were suspended in ethanol and sonicated; $5 \mu \mathrm{L}$ of this suspension was deposited on a copper grid (300 mesh) coated with holey carbon film. The copper grids were allowed to dry in air.

Nanophosphors deposited on silicon wafers, both as-deposited and fixed, were analysed by a Zeiss SIGMA VP field emission gun-scanning electron microscopy (FEG-SEM), using the in-lens secondary electron (Everhart-Thornley) detector.

A Vilber Lourmat UV lamp was used on the luminescent films, both as-deposited and fixed, selecting a wavelength of $254 \mathrm{~nm}$.

Photoluminescence excitation (PLE) and emission (PL) measurements were carried out using a Horiba-Jobin Yvon Fluorolog 3-21 spectrofluorimeter. A Xenon arc lamp was used as a continuous-spectrum source selecting the excitation wavelength by a double Czerny-Turner monochromator. The detection system was constituted by an iR300 single grating monochromator coupled to an R928 photomultiplier tube operating at $950 \mathrm{~V}$. The excitation spectra were recorded in the 250-450 nm range with $1 \mathrm{~nm}$ bandpass resolution, dividing the PMT signal by the intensity of the lamp, measured by using a calibrated photodetector. On the other hand, the emission spectra were recorded in the 550-750 $\mathrm{nm}$ range with $1 \mathrm{~nm}$ band-pass resolution and corrected for the response of the instrument.

Coating thickness measurements were performed by spectroscopic ellipsometry by using a J. A. Woollam variable angle V-VASE model operating in reflection at $50^{\circ}$ from vertical in the range $400-800 \mathrm{~nm}$. The analysis of the experimental data was done by using an Effective Medium Approximation (EMA) model describing the system as a single layer combination of $\mathrm{GdVO}_{4}$ nanocrystals and voids, with or without silica, using tabulated index of refraction. The fitting was performed by WVASE $®$ ellipsometric analysis programme.

The fixing action of the silica-based coating layer was estimated by PL emission measurements performed before and after a Scotch ${ }^{\mathrm{TM}}$ magic tape (3M Co.) peeling test, while the scratch resistance of the fixed nanoparticles was performed using an Alpha-Step IQ surface profilometer (KLA Tencor), equipped with a contact stylus (radius of $2 \mu \mathrm{m}$ ) and applying a stylus tracking force of $2.94 \mathrm{mg}$. The scan length was $3000 \mu \mathrm{m}$ and the speed was $50 \mu \mathrm{m} / \mathrm{s}$.

\section{Results and discussion}

\subsection{Properties of the synthesized nanophosphor particles}

The X-ray diffraction pattern of the $\mathrm{Eu}^{3+}$ doped $\mathrm{GdVO}_{4}$ nanophosphors here synthesized confirmed the attainment of pure 


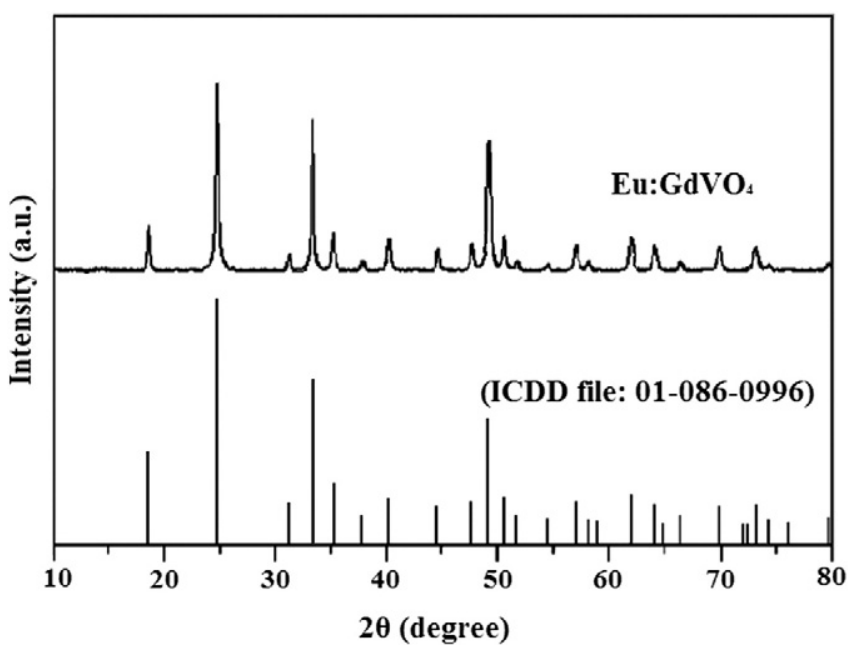

Fig. 1. X-ray diffraction profile of Eu-doped gadolinium orthovanadate crystals and the corresponding ICDD pattern.

gadolinium orthovanadate crystals with tetragonal structure (ICDD pattern no. 01-086-0996) (Fig. 1).

The nanophosphors appear as almost equiaxial nanocrystals with a mean size of $60 \mathrm{~nm}$ and a narrow distribution in size, as shown by the TEM micrographs reported in Fig. 2. Although the crystals were driven to agglomerate on the TEM grid during solvent evaporation, they are clearly well separated and do not form real agglomerates. This means that with appropriate dilution they might be deposited on a substrate with different degrees of coverage.

\subsection{Morphology of the coatings}

Fig. 3a-b shows a low-magnification FEG-SEM image of the deposition obtained by spray-coating ( 6 scans) using the Sol25 and Sol50 samples on a clean silicon substrate. It can be seen that both solutions leave uncovered areas, but that the coverage fraction is higher using Sol50. The amount of coverage is an important parameter for the fixing function of the layer deposited by plasma jet, since the coating molecules need to find uncovered areas where they can anchor on the substrate. Adhesion tests will tell whether the uncovered area is sufficient to guarantee a good anchoring of the fixing silica layer in both samples.

A FEG-SEM image of the Eu: $\mathrm{GdVO}_{4}$ nanocrystals at higher magnification (Sol50 as-deposited) is shown in Fig. 3c, and can be compared with the same sample coated with the fixing amorphous silica layer obtained by plasma jet coating (Sol50 fixed) reported in Fig. 3d. In the asdeposited sample, the regular and faceted shape of the nanocrystals may be clearly seen with their average size of about $60 \mathrm{~nm}$. Once coated, the cubic shape of the nanophosphor crystal was not observed. Instead, most particles showed a rather spherical appearance and a larger apparent size, due to the presence of thin silica layer evenly covering the surface (conformal growth). In Fig. 3e and $\mathrm{f}$ the cross-section images of the fixed sample are shown at two different magnifications and tilt angles giving an idea of the roughness of the surface. Comparing top-view and cross-section images it can be argued that the surface of both samples (Sol50 as-deposited and Sol50 fixed) is rough on the $100 \mathrm{~nm}$ scale, varying from zero to the size of clusters of few crystals, yielding to thickness of the order of few hundreds of $\mathrm{nm}$, in agreement with the ellipsometry measurements reported below.

FT-IR spectroscopy was employed to analyse the chemical structure of the fixing layer, deposited by APPJ technique, using hexamethyldisiloxane (HMDSO) as a precursor agent. Thanks to a dedicated heating system (at $125^{\circ} \mathrm{C}$ ), vapour of the organo-silane precursor flows inside the plasma nozzle where the precursor is chemically rearranged leading to the formation of a plasma polymerized film of amorphous $\mathrm{SiO}_{\mathrm{x}} \mathrm{C}_{\mathrm{y}} \mathrm{H}_{\mathrm{z}}$ on the surface of the treated material.

The infrared spectrum of the sample Sol50 fixed, reported in Fig. 4, shows intense bands between 800 and $1200 \mathrm{~cm}^{-1}$ and a broad band above $3000 \mathrm{~cm}^{-1}$. The highly intense peak at $1096 \mathrm{~cm}^{-1}$, attributed to the stretching of $\mathrm{Si}-\mathrm{O}$ bonds, confirms the presence of silica as coating. The shoulder between $1160 \mathrm{~cm}^{-1}$ and $1210 \mathrm{~cm}^{-1}$ due to the Si$\mathrm{O}$ bonds is attributed to the disorder-induced mechanical coupling of two asymmetric stretching vibrations in plasma-polymerized films [28]. The peak located around $938 \mathrm{~cm}^{-1}$ has been attributed to the stretching vibrations of $\mathrm{Si}-\mathrm{OH}$ groups and the broad band centred at around $3450 \mathrm{~cm}^{-1}$ has been assigned to the stretching frequency of free $\mathrm{O}-\mathrm{H}$ bonds of the silica coating. A small stretching absorption band, attributable to the $\mathrm{Si}\left(\mathrm{CH}_{3}\right)_{\mathrm{x}}$ group, is visible at $801 \mathrm{~cm}^{-1}$, attesting the slight organic character, of the obtained coating. No absorption signals were detected for the bare silicon wafer and the sample Sol50 asdeposited.

The thickness of the deposited layers was evaluated by spectroscopic ellipsometry, since the difference measured between the as-deposited and fixed samples can give an estimate of the silica conformal covering. Ellipsometry characterization of the luminescent layers, deposited and fixed on the surface of a Si wafer substrate, was realized in two steps. First, the thickness of the luminescent layer, obtained by spraydeposition of two different concentrations of nanophosphors in ethanol ( 0.25 wt.\% and $0.50 \mathrm{wt} . \%$ ) was evaluated. Then, the thickness of the layer on the same samples after fixing by APPJ technique was determined. In order to have an independent measure of the latter, a plasma jet deposition was made on a clean silicon wafer in the same operative conditions. Since the material is a multicomponent system constituted by almost spherical nanoparticles deposited on a silicon wafer, the effective medium approximation (EMA) [29] is the most appropriate
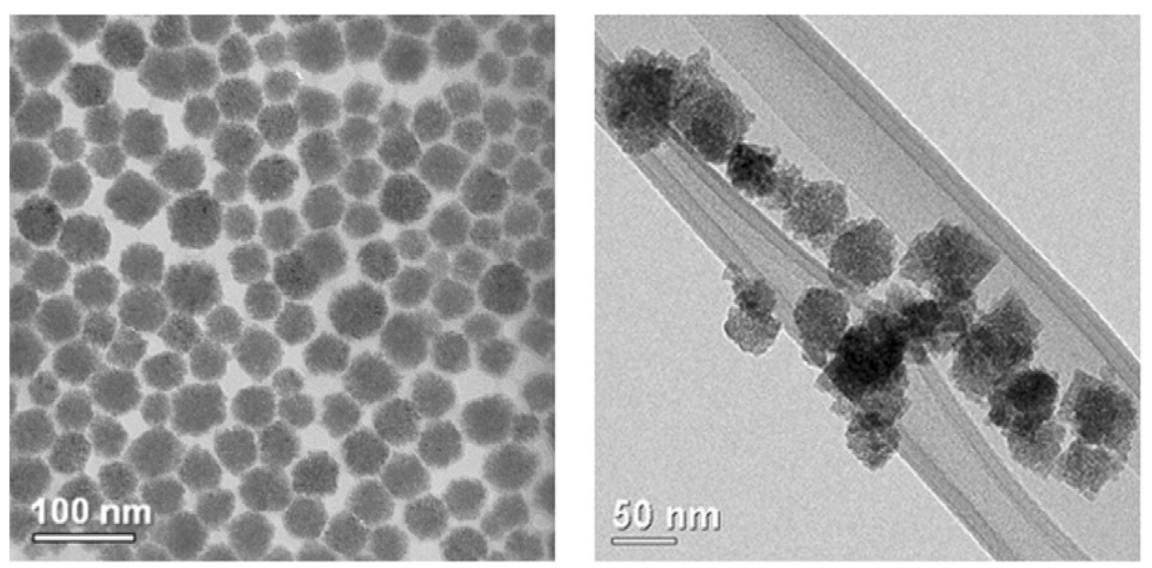

Fig. 2. Transmission electron micrographs of Eu: $\mathrm{GdVO}_{4}$ nanocrystals at two different magnifications. 

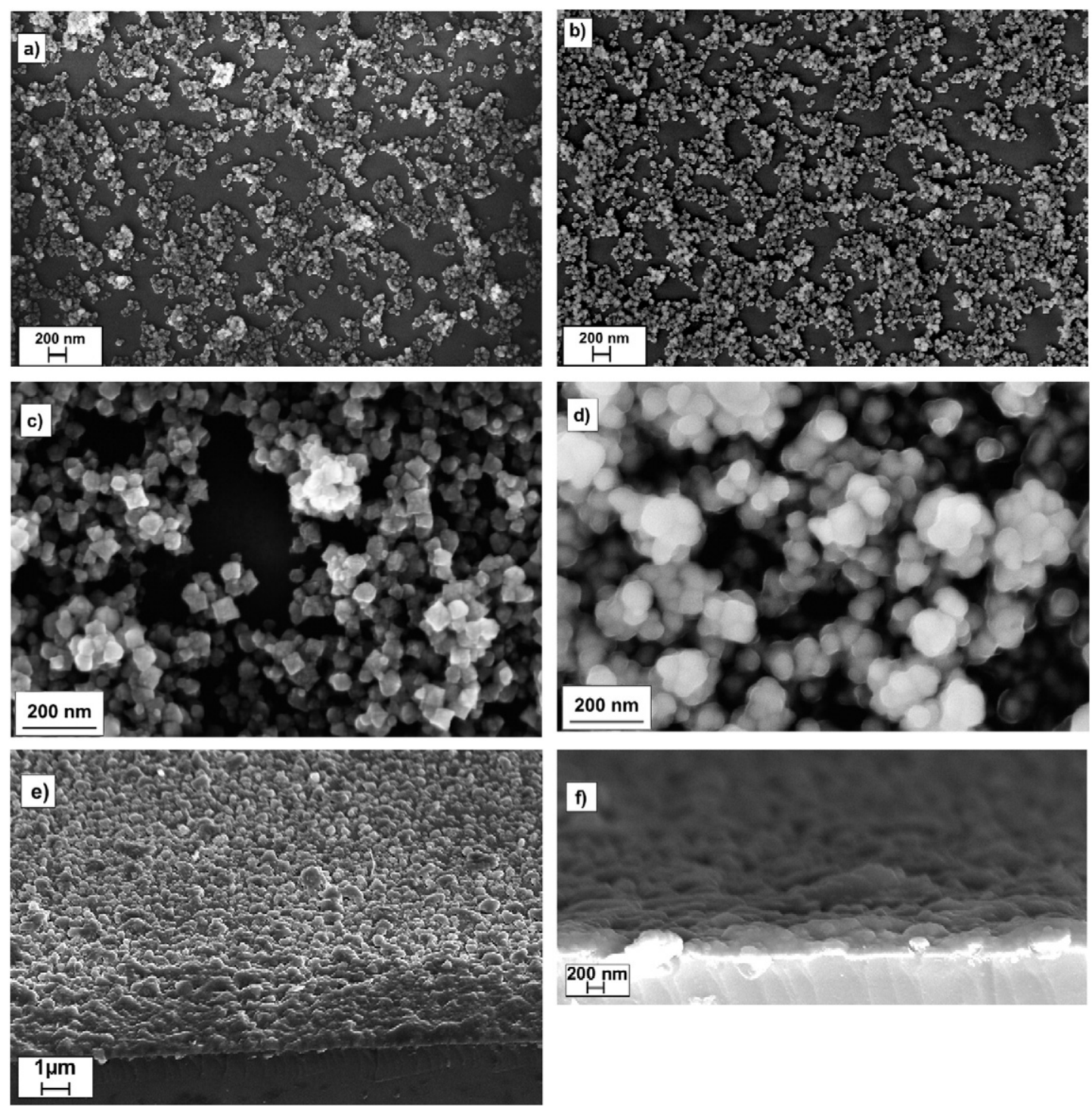

Fig. 3. FEG-SEM images of Eu $u^{3+}$-doped $\mathrm{GdVO}_{4}$ nanocrystals deposited on a silicon wafer. Low-magnification of a) Sol25 as-deposited and b) Sol50 as-deposited (by spray-deposition); Higher magnification of c) Sol50 as deposited and d) Sol50 fixed (by plasma jet deposition of an amorphous silica layer); e) and f) cross-section images of the Sol50 fixed sample at two different tilt angles and magnifications. 6 scans were used for the deposition, 4 scans for the fixing operation.

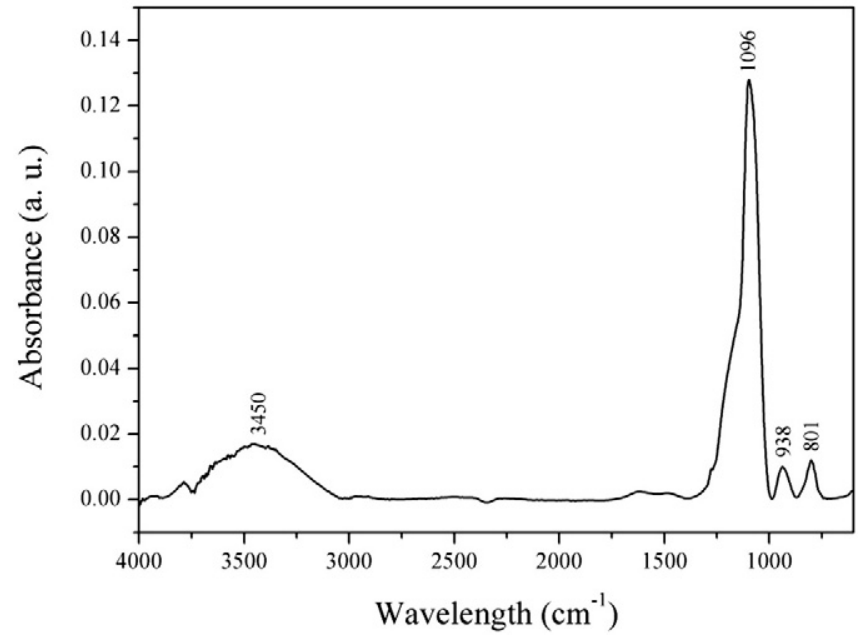

Fig. 4. FT-IR spectrum of the sample Sol50 fixed, obtained by spray deposition ( 6 scans) on a silicon wafer of a $0.50 \mathrm{wt}$.\% Eu: $\mathrm{GdVO}_{4}$ ethanol solution and by fixing ( 4 scans) the luminescent layer by a silica-based coating with APPJ technology. model to describe its dielectric properties. In particular the Bruggeman model [30] works well for spherical (or ellipsoidal) particles and describes the macroscopic dielectric function of the multicomponent material as a weight sum of the known dielectric functions of its components, giving the possibility to obtain the thickness and a porosity factor (fraction of void areas, if one component is given the refraction index of air). In our analysis we followed this approach, describing the system as a single layer combination of Eu: $\mathrm{GdVO}_{4}$ nanocrystals and voids, with or without silica. Table 1 is a summary of the measured values.

Table 1

Ellipsometry measurements of the thickness and PL emission intensity of the films, obtained starting from two different concentrations of the spray solution ( $0.25 \mathrm{wt} . \%$ and 0.50 wt.\%), as-deposited ( 6 scans) and fixed ( 4 scans) on monocrystalline silicon wafers.

\begin{tabular}{lll}
\hline Sample & $\begin{array}{l}\text { Film thickness } \\
(\mathrm{nm}) \pm 10 \%\end{array}$ & $\begin{array}{l}\text { PL intensity } \\
(\text { arb. units }) \pm 10 \%\end{array}$ \\
\hline Fixing layer & 37 & - \\
Sol25 as-deposited & 83 & 6.0 \\
Sol25 fixed & 120 & 5.8 \\
Sol50 as-deposited & 150 & 19 \\
Sol50 fixed & 190 & 20 \\
\hline
\end{tabular}



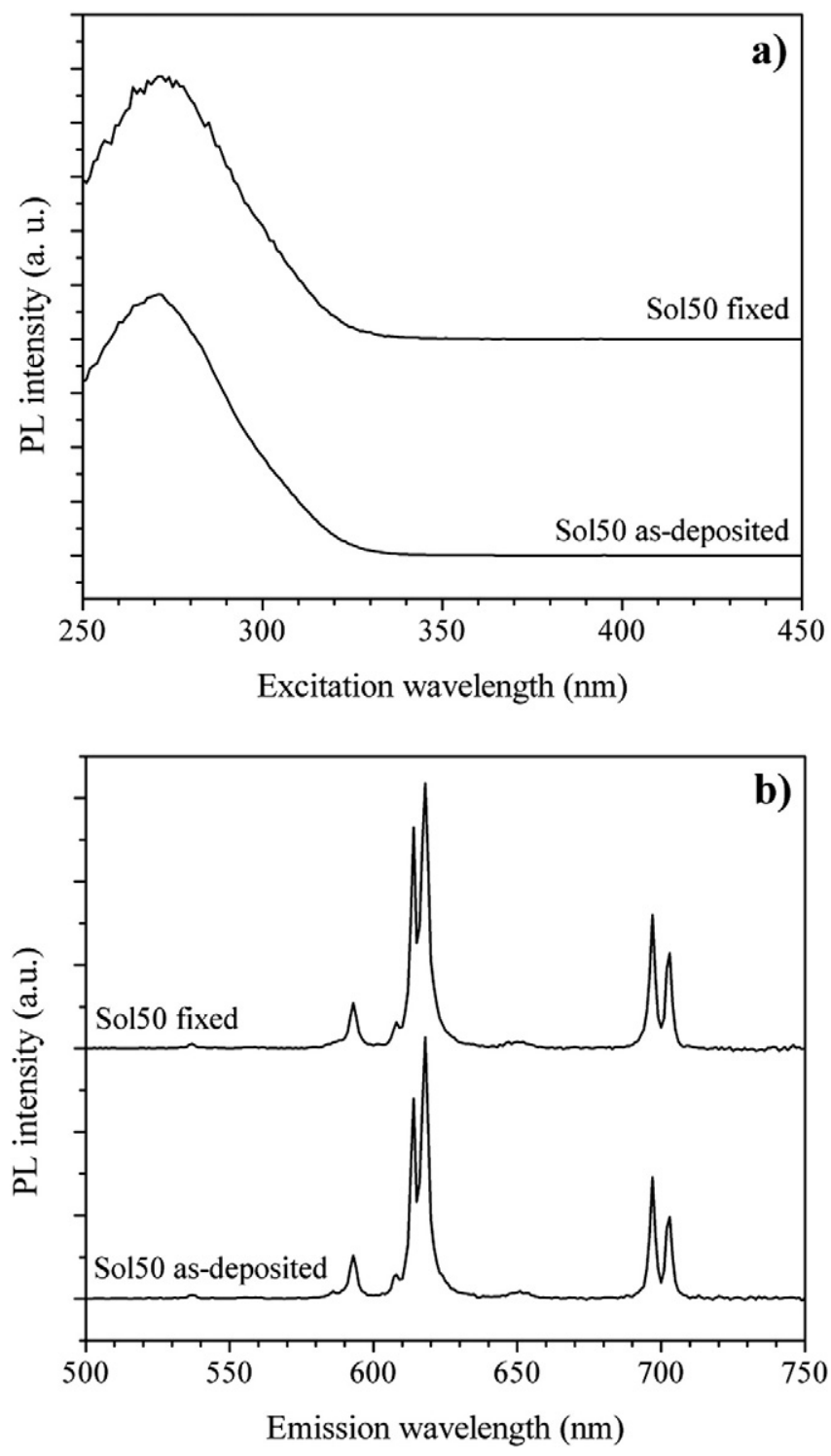

Fig. 5. a) PL excitation spectra $\left(\lambda_{\mathrm{em}}=618 \mathrm{~nm}\right)$ of the $\mathrm{Eu}^{3+}$-doped $\mathrm{GdVO}_{4}$ nanocrystals deposited on a silicon wafer: as-deposited ( 6 scans) and fixed ( 4 scans). b) PL emission spectra $\left(\lambda_{\text {ex }}=274 \mathrm{~nm}\right)$ of the nanophosphors deposited on a silicon wafer: as-deposited and fixed.

The thickness of the amorphous silica-based fixing layer, obtained by APPJ and starting from two different concentrations of the spray solution, was evaluated from the difference between the as-deposited and fixed samples and found about $40 \mathrm{~nm}$ for both samples (Table 1), in good agreement with the value obtained for the layer deposited on the clean silicon wafer.

\subsection{Optical properties of the coatings}

The luminescence properties of the Eu: $\mathrm{GdVO}_{4}$ nanocrystals, asdeposited and fixed on a silicon wafer, were first evaluated qualitatively by a Wood's lamp and then by photoluminescence measurements. In order to determine the optimum concentration of nanophosphors in solution to obtain a homogeneous spray deposition on the surface of the silicon substrate, several films were prepared from dispersions at 0.25 , 0.50 and 0.75 wt.\% of Eu: $\mathrm{GdVO}_{4}$ in ethanol. All samples showed a homogeneously distributed luminescence in the red range when illuminated by a UV lamp, but the intensity changed depending on the spray coating operating conditions used. The most intense luminescence was obtained when the concentration of the nanophosphors in ethanol was in the range 0.25 wt.\%-0.50 wt.\% while a higher concentration ( 0.75 wt.\%) did not lead to an increase of the intensity, rather a decrease. No visible difference in emission intensity was detected between as-deposited and fixed samples.

The samples were then characterized by photoluminescence spectroscopy and the spectra of samples Sol50 as-deposited and Sol50 fixed are reported as a representative example in Fig. 5. The spectra show the same features described in a previous detailed study of this nanophosphors, where attribution of the peaks and symmetry considerations can be found [26].

No significant difference can be observed between the two PLE excitation spectra (Fig. 5a) obtained before and after silica coating, indicating that the atmospheric plasma treatment has no effect on the excitation behaviour of $\mathrm{Eu}^{3+}$ doped nanocrystals.

The same can be said of the PL emission spectra. It can be also observed in Fig. 5b that the spectrum of the fixed sample (after atmospheric pressure plasma jet deposition of an amorphous silica layer) has the same shape and similar intensities as the one of the asprepared sample, indicating that the silica-based layer is transparent and that the plasma treatment has no effect on the $\mathrm{Eu}^{3+}$ surrounding environment, and consequently on the photoluminescence properties of the nanocrystals.

This technique also confirmed that a more intense photoluminescence was obtained when the concentration of Eu: $\mathrm{GdVO}_{4}$ in ethanol was in the range $0.25 \mathrm{wt} . \%-0.50 \mathrm{wt} . \%$, while a concentration of $0.75 \mathrm{wt} . \%$ led to a decrease of the intensity (not shown). We argued that for concentrations higher than $0.50 \mathrm{wt} . \%$, the nanophosphors in ethanol tended to agglomerate during the time of the process and precipitate, thus not flowing to the nozzle. In fact, a certain deposit was observed on the bottom of the syringe at the end of the spraying process for the concentration of $0.75 \mathrm{wt} . \%$, not allowing to quantify the real composition of the flow feeding the nozzle and therefore the real amount of nanophosphors deposited on the wafer. To state it more clearly, what actually changed with the concentration was the velocity of the sedimentation, which below 0.75 wt.\%
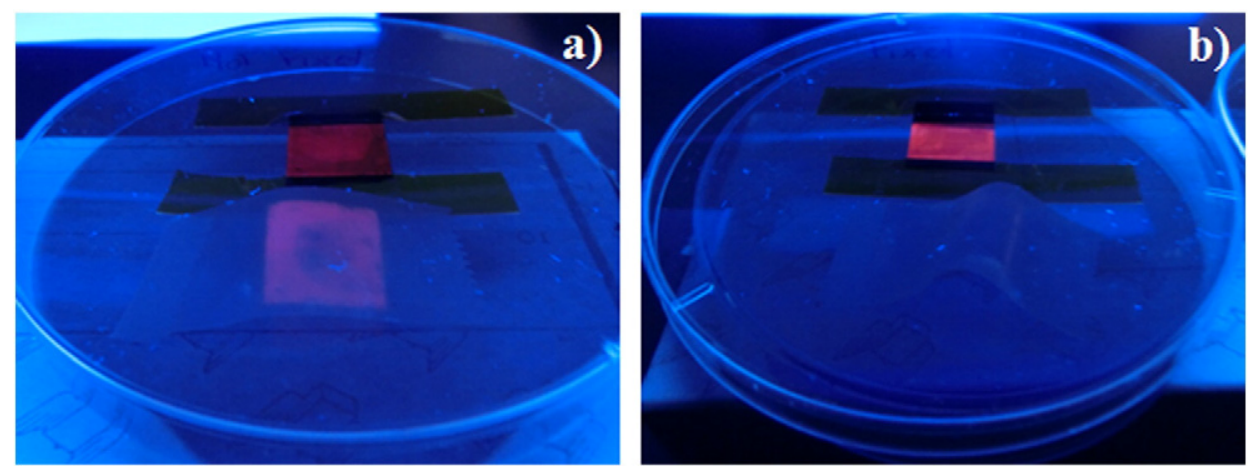

Fig. 6. Scotch tape peeling test visual results for sample Sol50: a) as-deposited (6 scans) and b) fixed (4 scans). 
Table 2

PL emission intensity of the samples Sol25 and Sol50, as-deposited (6 scans) and fixed (4 scans), before and after scotch tape peeling test. The PL relative intensity was estimated on the most intense emission peak (at $618 \mathrm{~nm}$ ), with an error of $\pm 10 \%$.

\begin{tabular}{|c|c|c|c|c|}
\hline \multirow{2}{*}{ Treatment } & \multicolumn{4}{|c|}{$\begin{array}{l}\text { PL relative intensity } \\
(\%)\end{array}$} \\
\hline & $\begin{array}{l}\text { Sol25 } \\
\text { as-deposited }\end{array}$ & $\begin{array}{l}\text { Sol25 } \\
\text { fixed }\end{array}$ & $\begin{array}{l}\text { Sol50 } \\
\text { as-deposited }\end{array}$ & $\begin{array}{l}\text { Sol50 } \\
\text { fixed }\end{array}$ \\
\hline Before scotch peeling & 100 & 100 & 100 & 100 \\
\hline After scotch peeling & 38 & 88 & 28 & 100 \\
\hline On the scotch tape & 59 & 2 & 70 & 0 \\
\hline
\end{tabular}

was slow enough to allow one to carry the spraying procedure before any deposit was observed, which was not the case for $0.75 \mathrm{wt}$.\%. In fact, for much longer times all solutions showed a deposit.

On the basis of these results, two concentrations of nanophosphors in ethanol were extensively investigated: 0.25 and $0.50 \mathrm{wt} . \%$ (samples Sol25 and Sol50, respectively).

It is worth observing that, in this range of concentration by doubling the concentration of the nanocrystal solution, the thickness also increases of about two times due to the higher amount of sprayed material (Table 1). On the other hand, the PL emission analysis, besides confirming that the PLintensity is not affected by the plasma deposition of the silica-based fixing layer, it reveals more than three-fold increase for the $0.50 \%$ concentrated sample with respect to the $0.25 \%$. In fact, not only is the thickness of the Sol50 samples larger, but, as shown in Fig. 3, the surface coverage is also higher for this sample, so that the total amount of luminescent material is increasing more than linearly.

In order to study the effect of multiple depositions of the nanocrystals on the wafer surface, a series of samples was prepared using an increasing number of spray treatments ( $4,6,8$ scans), keeping the concentration of nanophosphors constant at a value of $0.50 \mathrm{wt} . \%$ in ethanol (Sol50 samples). It was observed that the spectra (not shown) maintained the same lines and shape for all of the samples, while the peak intensities changed depending to the number of scans, yet not in a linear way. Both PLE and PL spectra revealed that 4 spray treatments gave rise to a low signal, most probably due to a low amount of luminescent particles deposited, but, surprisingly, also 8 spray treatments led to the same result, with a low intensity of luminescence. We then argued that the pressure of the spray was removing part of the nanophosphors deposited during the previous scan: in fact, as also visually observed, the higher the film thickness the lower its cohesion with the substrate. Apparently, the highest number of deposited particles is the result of a trade off between adhesion force and gas flux. With the gas flux used, the 6 spray treatments led to the highest PLE and PL signal, so this parameter was chosen and used for the subsequent tests.

\subsection{Adhesion tests}

Finally, in order to verify the ability of the silica-based film to effectively act as a fixing layer, as-deposited and fixed samples ( 0.25 and 0.50 wt.\% solutions, choosing 6 scans as a spray treatment) were tested for adhesion by applying a scotch tape peeling test. Scotch ${ }^{\mathrm{TM}}$ magic tape (3 M Co.) was used for this purpose. Images of Fig. 6, obtained under an UV lamp, show the test for the sample Sol50, taken as an example. After test on the as-deposited sample (Fig. 6a), the luminescent particles were easily removed and their luminescent activity was clearly visible on the tape surface, whereas for the fixed sample (Fig. 6b) the luminescent activity remained unchanged on the silicon wafer surface, and the scotch tape surface did not show any luminescence. Very similar results were obtained for sample Sol25, as-deposited and fixed.

The results obtained by measuring the PL emission intensity of the un-pealed and pealed samples Sol25 and Sol50, both as-deposited and fixed, are shown in Table 2, quantitatively confirming that most of the luminescence signal is preserved in the silica coated sample, with negligible intensity on the scotch tape surface for sample Sol25 fixed, whereas only $40 \%$ of the signal is retained in the sample without silica protection (Sol25 as-deposited) and 60\% of the initial signal is measured on the scotch tape. The results are even better when the loading of nanophosphors deposited has been doubled (Sol50): the luminescence signal on the silicon wafer surface is completely retained after scotch tape peeling test (Table 2 ).

As-deposited and fixed samples were also compared under the action of a stylus profilometer that acts as a hardness test, by applying a stylus tracking force of $2.94 \mathrm{mg}$ for a scan length $3 \mathrm{~mm}$. Results of Fig. 7 show that, after a stylus scan, the as-deposited sample Sol50 presents a scratch line, while fixed sample appears perfectly intact, thus confirming the real fixing action of the silica-based coating.

The effectiveness of the fixing ability of the Eu-doped $\mathrm{GdVO}_{4}$ nanophosphors by a $40 \mathrm{~nm}$ thick silica-based layer is being tested with good results on different stone materials for applications for anticounterfeiting of artworks and the possibility of patterning the deposit is being evaluated.

\section{Conclusions}

The possibility to obtain a well-adhered highly luminescent, transparent thin layer of efficient $\mathrm{Eu}^{3+}$-doped gadolinium orthovanadate nanophosphors on monocrystalline silicon wafers using atmospheric pressure plasma jet was investigated. The role of the different parameters used was elucidated and the optimized working conditions were determined. A study of the operative conditions of the spray procedure established that using 6 scans (subsequent treatments) and a concentration of Eu: $\mathrm{GdVO}_{4}$ nanocrystals in ethanol in the range 0.25-0.50 wt.\% yielded the best performances. A very high luminescence emission and well adhered layer were obtained by depositing a silica-based film of
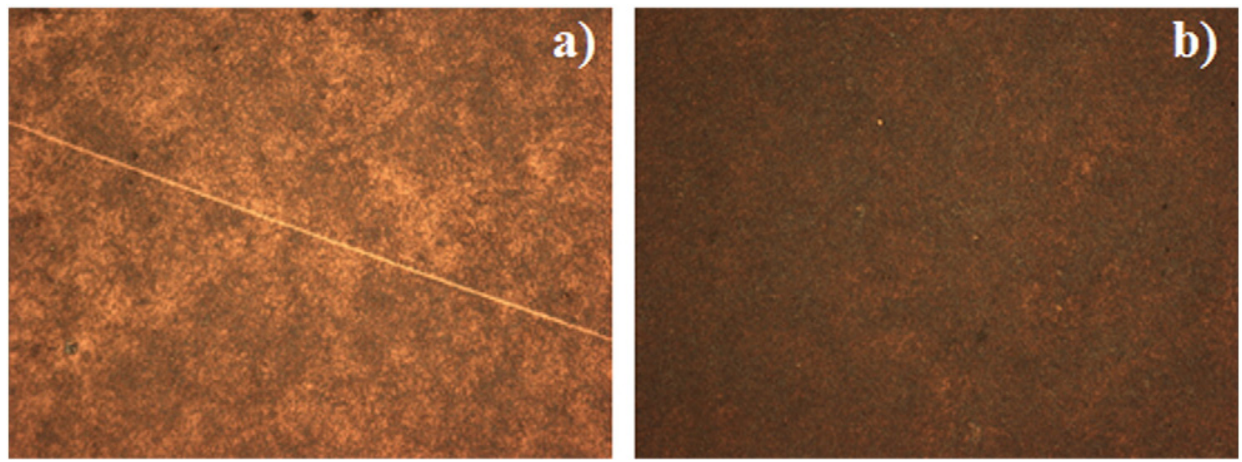

Fig. 7. Photomicrographs obtained at $50 \times$ after the scratch test for samples: a) Sol50 as-deposited ( 6 scans) and b) Sol50 fixed (4 scans). 
about $40 \mathrm{~nm}$ thick, which fixed the luminescent layer previously sprayed, ensuring mechanical protection to the nanoparticles even after a scotch tape peeling test and a scratch test with a stylus profilometer. The results pointed out that the plasma deposition process and the silica-based coating did not damage the nanophosphors, preserving on the contrary their photoluminescence emission properties.

In summary a luminescent film, transparent and undetectable in the presence of visible light but easily activated by UV light source, displaying good mechanical properties, was successfully obtained on the surface of a silicon substrate. The encouraging results indicate that the proposed methodology could be very interesting for its applicability on different substrates and optically active coatings could be therefore easily realized by using the technology here described.

\section{Acknowledgements}

Università Ca' Foscari Venezia and Consortium INSTM are acknowledged for the financial support.

\section{References}

[1] R. Kubrin, Nanophosphors coatings: technology and applications, opportunities and challenges, Kona Powder Part. J. 31 (2014) 22-52.

[2] E. Moretti, A. Talon, L. Storaro, A. Le Donne, S. Binetti, A. Benedetti, S. Polizzi, Concentration quenching and photostability in $\mathrm{Eu}(\mathrm{dbm})_{3}$ phen embedded in mesoporous silica nanoparticles, J. Lumin. 146 (2014) 178-185.

[3] F.C.J.M. van Veggel, C. Dong, N.J.J. Johnson, J. Pichaandi, $\operatorname{Ln}\left({ }^{3+}\right)$-doped nanoparticles for upconversion and magnetic resonance imaging: some critical notes on recent progress and some aspects to be considered, Nanoscale 4 (2012) 7309-7321.

[4] B.M. Tissue, Synthesis and luminescence of lanthanide ions in nanoscale insulating hosts, Chem. Mater. 10 (1998) 2837-2845.

[5] B.K. Gupta, V. Rathee, T.N. Narayan, P. Thanikaivelan, A. Saha, S.P. Singh, V. Shanker, A.A. Narti, P.M. Alayan, Probing a bifunctional luminomagnetic nanophosphor for biological applications: a photoluminescence and time-resolved spectroscopic study, Small 7 (2011) 1767.

[6] K.Y. Sasaki, J.B. Talbot, Deposition of powder phosphors for information displays, Adv. Mater. 11 (1999) 91-105.

[7] Y. Tomita, Coating methods. methods of phosphor synthesis and related technology, in: W.M. Yen, S. Shionoya, H. Yamamoto (Eds.), Phosphor Handbook, second ed.CRC Press, Boca Raton, Fla. 2007, pp. 399-400.

[8] E. Sluzky, K. Hesse, Electrophoretic preparation of phosphor screens, J. Electrochem. Soc. 136 (1989) 2724-2727.

[9] J.-H. Yum, S.-Y. Seo, S. Lee, Y.-E. Sung, $\mathrm{Y}_{3} \mathrm{~A}_{15} \mathrm{O}_{12}: \mathrm{Ce}_{0.05}$ phosphor coatings on gallium nitride for white light emitting diodes, J. Electrochem. Soc. 150 (2003) H47-H52.

[10] R. Fujiwara, H. Sano, S. Harada, M. Shimizu, H. Takebe, M. Kuwabara, Fabrication of electroluminescent devices using nanocrystalline oxide phosphors, Key Eng. Mater. 388 (2008) 123-126.

[11] B. Fleury, G. Dantelle, S. Darbe, J.-P. Boilot, T. Gacoin, Transparent coatings made from spray deposited colloidal suspensions, Langmuir 28 (2012) 7639-7645.
[12] A. Revaux, G. Dantelle, S. Brinkley, E. Matioli, C. Weisbuch, J.-P. Boilot, T. Gacoin, YAG:Ce nanoparticle based converter layer for white LEDs, Proc. SPIE 8102 (2011) 81020R.

[13] M.L. Pang, J. Lin, Z.Y. Cheng, J. Fu, R.B. Xing, S.B. Wang, Patterning and luminescent properties of nanocrystalline $\mathrm{Y}_{2} \mathrm{O}_{3}$ : $\mathrm{Eu}_{3}^{+}$phosphor films by sol-gel soft lithography, Mater. Sci. Eng. B 100 (2003) 124-131.

[14] Y.C. Wu, C. Garapon, R. Bazzi, A. Pillonnet, O. Tillement, J. Mugnier, Optical and fluorescent properties of $\mathrm{Y}_{2} \mathrm{O}_{3}$ sol-gel planar waveguides containing $\mathrm{Tb}^{3+}$ doped nanocrystals, Appl. Phys. A Mater. Sci. Process. 87 (2007) 697-704.

[15] V. Bedekar, D.P. Dutta, A.K. Tyagi, White light emission from spin coated $\mathrm{Gd}_{2} \mathrm{O}_{3}: \mathrm{dy}$ nano phosphors synthesized using polyol technique, J. Nanosci. Nanotechnol. 10 (2010) 8234-8238

[16] V. Buissette, D. Giaume, T. Gacoin, J.-P. Boilot, Aqueous routes to lanthanide-doped oxide nanophosphors, J. Mater. Chem. 16 (2006) 529-539.

[17] C.K. Huang, Y.C. Chen, W.B. Hung, T.M. Chen, K.W. Sun, W.-L. Chang, Enhanced ligh harvesting of $\mathrm{Si}$ solar cells via luminescent down-shifting using $\mathrm{YVO} 4: \mathrm{Bi}^{3+}, \mathrm{Eu}^{3+}$ nanophosphors, Prog. Photovolt. Res. Appl. 21 (2012) 1507-1513.

[18] A.F. Khan, D. Haranath, R. Yadav, S. Singh, S. Chawla, V. Dutta, Controlled surface distribution and luminescence of $\mathrm{YVO}_{4}: \mathrm{Eu}^{3+}$ nanophosphor layers, Appl. Phys. Lett. 93 (2008) 073103.

[19] A. Klausch, H. Althues, T. Freudenberg, S. Kaskel, Wet chemical preparation of $\mathrm{YVO}_{4}$ : Eu thin films as red-emitting phosphor layers for fully transparent flat dielectric discharge lamp, Thin Solid Films 520 (2012) 4297-4301.

[20] P. Psuja, D. Hreniak, W. Strek, D. Kovalenko, V. Gaishun, Fabrication and properties of nanocomposite ITO layer containing terbium doped yttrium aluminum garnet nanocrystallites, Proc. SPIE 7212 (2009) 72121C.

[21] A. Revaux, G. Dantelle, D. Decanini, A.-M. Haghiri-Gosnet, T. Gacoin, J.-P. Boilot, Synthesis of YAG:Ce/ $/ \mathrm{TiO}_{2}$ nanocomposite films, Opt. Mater. 33 (2011) 1124-1127.

[22] W.-S. Song, Y.-S. Kim, H. Yang, Construction of highly transparent plasma display devices using hydrothermally synthesized green-emitting $\mathrm{LaPO}_{4}: \mathrm{Ce}, \mathrm{Tb}$ nanophosphors, J. Electrochem. Soc. 158 (2011) J137-J142.

[23] S.B. Fuller, E.J. Wilhelm, J.M. Jacobson, Ink-jet printed nanoparticle microelectromechanical systems, IEEE ASME J. Microelectron. Syst. 11 (2002) 54-60.

[24] Y. Lu, G.L. Liu, L.P. Lee, High-density silver nanoparticle film with temperaturecontrollable interparticle spacing for a tunable surface enhanced Raman scattering substrate, Nano Lett. 5 (2005) 5-9.

[25] A. Beggio, M. Fantin, P. Scopece, A. Surpi, A. Patelli, A. Benedetti, D. Cristofori, F. Enrichi, Incorporation of Eu-Tb codoped nanophosphors in silica-based coatings assisted by atmospheric pressure plasma jet technology, Thin Solid Films 578 (2015) 38-48.

[26] N.O. Nuñez, S. Rivera, D. Alcantara, J.M. de la Fuente, J. García-Sevillano, M. Ocaña Surface modified Eu:GdVO $\mathrm{G}_{4}$ nanocrystals for optical and MRI imaging, Dalton Trans. 42 (2013) 10725-10734.

[27] A. Mardegan, V. Pifferi, E. Pontoglio, L. Falciola, P. Scopece, L.M. Moretto, Sprayed carbon nanotubes on pyrolysed photoresist carbon electrodes: application to o-toluidine determination, Electrochem. Commun. 48 (2014) 13-16.

[28] R. Förch, A.N. Chifen, A. Bousquet, L.H. Khor, M. Jungblut, L.-Q. Chu, Z. Zhang, I. OseyMensah, E.-K. Sinner, W. Knoll, Recent and expected roles of plasma-polymerized films for biomedical applications, Chem. Vap. Depos. 13 (2007) 280-294.

[29] M. Wang, N. Pan, Predictions of effective physical properties of complex multiphase materials, Mater. Sci. Eng. R 63 (2008) 1-30.

[30] D.A.G. Bruggeman, Berechnung verschiedener physikalischer konstanten von heterogenen substanzen, Ann. Phys. 416 (1935) 636-679. 